\title{
Theory of Defect Dynamics in Graphene
}

\author{
L.L. Bonilla ${ }^{1}$ and A. Carpio ${ }^{2}$ \\ ${ }^{1}$ Universidad Carlos III de Madrid \\ ${ }^{2}$ Universidad Complutense de Madrid \\ Spain
}

\section{Introduction}

The experimental discovery of graphene (single monolayers of graphite) (Novoselov et al, 2004; 2005) and its extraordinary properties due to the Dirac-like spectrum of its charge carriers have fostered an enormous literature reviewed by a number of authors (Castro Neto et al., 2009; Geim \& Novoselov, 2007; Geim, 2009; Vozmediano et al., 2010). The electronic, chemical, thermal and mechanical properties of graphene are exceptionally sensitive to lattice imperfections (Castro Neto et al., 2009; Geim \& Novoselov, 2007). These defects and even the ripples that always cover suspended graphene sheets (Fasolino et al., 2007; Meyer et al., 2007) induce pseudo-magnetic gauge fields (Vozmediano et al., 2010) and have given rise to the notion of strain engineering (Guinea et al., 2009). Thus the study of defects and ripples in graphene is crucial and it has generated important experimental work (Coleman et al., 2008; Gómez-Navarro et al., 2010; Meyer et al., 2008; Wang et al., 2008). Real time observation of defect dynamics is possible using Transmission Electron Microscopes (TEM) corrected for aberration that have single atom resolution (Meyer et al., 2008). In these studies, defects are induced by irradiation and their evolution is observed in a time scale of seconds (Meyer et al., 2008), much longer than sub-picosecond time scales typical of sound propagation in a primitive cell.

The graphene lattice is hexagonal and defects appear in different forms: pentagon-heptagon (5-7) pairs, Stone-Wales (SW) defects (two adjacent pentagon-heptagon pairs in which the heptagons share one side, denoted in short as 5-7-7-5 defects) (Meyer et al., 2008), pentagon-octagon-pentagon (5-8-5) divacancies (Coleman et al., 2008), asymmetric vacancies (nonagon-pentagon or 9-5 pairs) and more complicated groupings such as 5-7-7-5 and 7-5-5-7 adjacent pairs or defects comprising three pentagons, three heptagons and one hexagon (Meyer et al., 2008). In other two dimensional (two-dimensional) crystals such as Boron Nitride (hBN) symmetric vacancies in which one atom is missing have been observed (Meyer et al., 2009). On the long time scale of seconds and for unstressed graphene, Stone-Wales defects are unstable: their two pentagon-heptagon pairs glide towards each other and annihilate, and the same occurs to defects comprising three pentagons, three heptagons and one hexagon, whereas 5-7-7-5 and 7-5-5-7 adjacent pairs remain stable (Meyer et al., 2008). In stressed graphene oxide samples, SW defects split into their component 5-7 pairs which then move apart (Gómez-Navarro et al., 2010). While most theoretical studies on the influence of defects in electronic properties assume a given defect configuration and then proceed to 
analyze its effects (Castro Neto et al., 2009; Vozmediano et al., 2010), it is important to predict defect stability and evolution.

In recent work, we have explained the observed long time defect dynamics in graphene by considering defects as the core of edge dislocations or dislocation dipoles in a planar two-dimensional hexagonal lattice (Carpio \& Bonilla, 2008; Carpio et al., 2008). Our theory is a computationally efficient alternative to ab initio approaches such as molecular dynamics or density functional theory (Abedpour et al., 2007; Fasolino et al., 2007; Segall, 2002; Thompson-Flagg et al., 2009). Our top-down approach starts from linear elasticity. We discretize continuum linear elasticity on a hexagonal lattice and replace differences of vector displacements along primitive directions by periodic functions thereof which are linear for small differences. Our periodized discrete elasticity allows dislocation gliding along primitive directions and it reduces to continuum linear elasticity very far from dislocation cores (Carpio \& Bonilla, 2005). Introducing a large damping in the resulting equations of motion and solving them numerically, we are able to predict the stable cores corresponding to a given dislocation configuration. Using this theory, we have predicted the stability of pentagon-heptagon defects (that are the cores of dislocations) (Carpio \& Bonilla, 2008; Carpio et al., 2008). Similarly, a study of dislocation dipoles in unstressed samples (Carpio \& Bonilla, 2008; Carpio et al., 2008) predicts that SW are unstable whereas symmetric vacancies, divacancies and 7-5-5-7 defects are stable. In stressed samples, our theory predicts that Stone-Wales defects split into two 5-7 pairs that move apart (Carpio \& Bonilla, 2008), as confirmed later by experiments (Gómez-Navarro et al., 2010).

In this chapter, we first describe periodized discrete elasticity for a planar graphene sheet and explain the evolution of several defects considered as cores of dislocations or dislocation dipoles. We then explain how an extension of our theory may describe a suspended graphene sheet in three dimensions, which is able to bend away from the planar configuration. We also discuss how to incorporate a mechanism for the formation and evolution of ripples. The rest of the chapter is as follows. Periodized discrete elasticity and its equations of motion are explained in Section 2 for a planar graphene sheet. The stable cores corresponding to the far field of a single edge dislocation and a single dislocation dipole are used in Section 3 to illustrate the way defects are constructed numerically. Our results are also compared to available experiments in graphene and other two-dimensional crystals, in particular to those by Meyer et al. (2008). Section 4 contains the extension to three space dimensions. We assume that the suspended sheet has a trend to locally bend upwards or downwards represented by an Ising spin. These spins are coupled to the carbon atoms in the sheet, are in contact with a thermal bath and evolve stochastically according to Glauber dynamics. Damping is caused by coupling to the bath and by Glauber dynamics. The formation of ripples in suspended graphene sheet is explained as a phase transition from the planar sheet that occurs below a certain critical temperature. Numerical solutions of the equations of motion illustrate the theoretical results and in particular show ripples and curvature of the sheet near a pentagon-heptagon defect. The last section is devoted to our conclusions.

\section{Periodized discrete elasticity of planar graphene}

In the continuum limit, small elastic deformations of graphene sheets have a free energy

$$
F_{g}=\frac{\kappa}{2} \int\left(\nabla^{2} w\right)^{2} d x d y+\frac{1}{2} \int\left(\lambda u_{i i}^{2}+2 \mu u_{i k}^{2}\right) d x d y,
$$


corresponding to a membrane (Nelson, 2002), in which $u_{i k}, \mathbf{u}=\left(u_{1}, u_{2}\right)=(u(x, y), v(x, y))$, $w(x, y), \kappa, \lambda=C_{12}$ and $\mu=C_{66}$ are the in-plane linearized deformation tensor, the in-plane displacement vector, the vertical deflection of the membrane, the bending stiffness (measured in units of energy) and the Lamé coefficients (Landau \& Lifshitz, 1986) of graphene (graphite is isotropic in its basal plane so that $\left.C_{11}=\lambda+2 \mu\right)$, respectively. We have used the convention of sum over repeated indices. In (1), $\nabla^{2} w=\partial_{x}^{2} w+\partial_{y}^{2} w$ is the two-dimensional laplacian and the two-dimensional Lamé coefficients have units of energy per unit area. Dividing by the distance between graphene planes in graphite, $0.335 \mathrm{~nm}$, they can be converted to the usual units for 3D graphite. The in-plane linearized deformation tensor is

$$
u_{i k}=\frac{1}{2}\left(\partial_{x_{k}} u_{i}+\partial_{x_{i}} u_{k}+\partial_{x_{i}} w \partial_{x_{k}} w\right), \quad i, k=1,2,
$$

in which we have ignored the small in-plane nonlinear terms $\partial_{x_{i}} u \partial_{x_{k}} u+\partial_{x_{i}} v \partial_{x_{k}} v$.

In this section, we shall consider that only in-plane deformations are possible so that $w=$ 0 . Then the equations of motion derived from (1)-(2) are the Navier equations of linear elasticity for $(u, v)$ (Landau \& Lifshitz, 1986). If we add to these equations a phenomenological damping with coefficient $\gamma$ (to be fitted to experiments), we have

$$
\begin{aligned}
\rho_{2} \partial_{t}^{2} u+\gamma \partial_{t} u & =(\lambda+2 \mu) \partial_{x}^{2} u+\mu \partial_{y}^{2} u+(\lambda+\mu) \partial_{x} \partial_{y} v, \\
\rho_{2} \partial_{t}^{2} v+\gamma \partial_{t} v & =\mu \partial_{x}^{2} v+(\lambda+2 \mu) \partial_{y}^{2} v+(\lambda+\mu) \partial_{x} \partial_{y} u,
\end{aligned}
$$

where $\rho_{2}$ is the two-dimensional mass density. The governing equations of our theory are obtained from (3)-(4) in a three step process (Carpio \& Bonilla, 2008): (i) discretize the equations on the hexagonal graphene lattice, (ii) rewrite the discretized equations in primitive coordinates, and (iii) replace finite differences appearing in the equations by periodic functions thereof in such a way that the equations remain invariant if we displace the atoms one step along any of the primitive directions. The last step allows dislocation gliding.

\subsection{Discrete elasticity}

Let us assign the coordinates $(x, y)$ to the atom $A$ in sublattice 1 (see Figure 1 ). The origin of coordinates, $(0,0)$, is also an atom of sublattice 1 at the center of the graphene sheet. The three nearest neighbors of $A$ belong to sublattice 2 and their cartesian coordinates are $n_{1}, n_{2}$ and $n_{3}$ below. Its six next-nearest neighbors belong to sublattice 1 and their cartesian coordinates are $n_{i}, i=4, \ldots, 9$ :

$$
\begin{aligned}
& n_{1}=\left(x-\frac{a}{2}, y-\frac{a}{2 \sqrt{3}}\right), n_{2}=\left(x+\frac{a}{2}, y-\frac{a}{2 \sqrt{3}}\right), n_{3}=\left(x, y+\frac{a}{\sqrt{3}}\right), \\
& n_{4}=\left(x-\frac{a}{2}, y-\frac{a \sqrt{3}}{2}\right), n_{5}=\left(x+\frac{a}{2}, y-\frac{a \sqrt{3}}{2}\right), n_{6}=(x-a, y), \\
& n_{7}=(x+a, y), n_{8}=\left(x-\frac{a}{2}, y+\frac{a \sqrt{3}}{2}\right), n_{9}=\left(x+\frac{a}{2}, y+\frac{a \sqrt{3}}{2}\right) .
\end{aligned}
$$

In Fig. 1, atoms $n_{6}$ and $n_{7}$ are separated from $A$ by the primitive vector $\pm \mathbf{a}$ and atoms $n_{4}$ and $n_{9}$ are separated from $A$ by the primitive vector $\pm \mathbf{b}$. Instead of choosing the primitive vector 


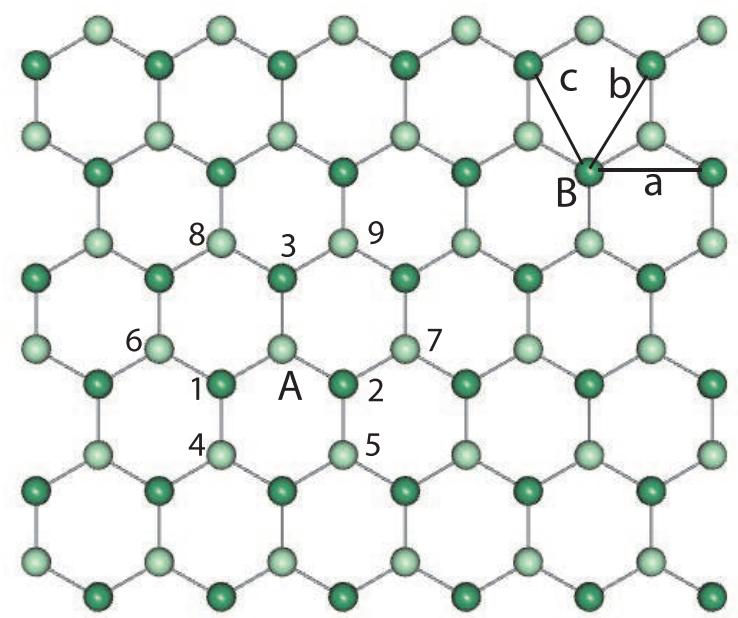

Fig. 1. Neighbors of a given atom $A$ in sublattice 1 (light colored atoms).

$\pm \mathbf{b}$, we could have selected the primitive direction $\pm \mathbf{c}$ along which atoms $n_{8}, A$ and $n_{5}$ lie. Let us define the following operators acting on functions of the coordinates $(x, y)$ of node $A$ :

$$
\begin{aligned}
T u & =\left[u\left(n_{1}\right)-u(A)\right]+\left[u\left(n_{2}\right)-u(A)\right]+\left[u\left(n_{3}\right)-u(A)\right] \sim\left(\partial_{x}^{2} u+\partial_{y}^{2} u\right) \frac{a^{2}}{4}, \\
H u & =\left[u\left(n_{6}\right)-u(A)\right]+\left[u\left(n_{7}\right)-u(A)\right] \sim\left(\partial_{x}^{2} u\right) a^{2}, \\
D_{1} u & =\left[u\left(n_{4}\right)-u(A)\right]+\left[u\left(n_{9}\right)-u(A)\right] \sim\left(\frac{1}{4} \partial_{x}^{2} u+\frac{\sqrt{3}}{2} \partial_{x} \partial_{y} u+\frac{3}{4} \partial_{y}^{2} u\right) a^{2}, \\
D_{2} u & =\left[u\left(n_{5}\right)-u(A)\right]+\left[u\left(n_{8}\right)-u(A)\right] \sim\left(\frac{1}{4} \partial_{x}^{2} u-\frac{\sqrt{3}}{2} \partial_{x} \partial_{y} u+\frac{3}{4} \partial_{y}^{2} u\right) a^{2},
\end{aligned}
$$

as the lattice constant $a$ tends to zero. Similar operators can be defined if we replace the point $A$ in sublattice 1 by a point belonging to the sublattice 2 . Now we replace in (3) and (4), $H u / a^{2},(4 T-H) u / a^{2}$ and $\left(D_{1}-D_{2}\right) u /\left(\sqrt{3} a^{2}\right)$ instead of $\partial_{x}^{2} u, \partial_{y}^{2} u$ and $\partial_{x} \partial_{y} u$, respectively, with similar substitutions for the derivatives of $v$, thereby obtaining the following equations at each point of the lattice:

$$
\begin{aligned}
& \rho_{2} a^{2} \partial_{t}^{2} u+\gamma \partial_{t} u=4 \mu T u+(\lambda+\mu) H u+\frac{\lambda+\mu}{\sqrt{3}}\left(D_{1}-D_{2}\right) v, \\
& \rho_{2} a^{2} \partial_{t}^{2} v+\gamma \partial_{t} v=4(\lambda+2 \mu) T v-(\lambda+\mu) H v+\frac{\lambda+\mu}{\sqrt{3}}\left(D_{1}-D_{2}\right) u .
\end{aligned}
$$

These equations have two characteristics time scales, the time $t_{s}=\sqrt{\rho_{2} a^{2} /(\lambda+2 \mu)}$ it takes a longitudinal sound wave to traverse a distance $a$, and the characteristic damping time, $t_{d}=\gamma a^{2} /(\lambda+2 \mu)$. Using the known values of the Lamé coefficients (Lee et al., 2008; Zakharchenko et al., 2009), $t_{s} \approx 10^{-14} \mathrm{~s}$. Our simulations show that it takes $0.4 t_{d}$ a SW to 
disappear after it is created by irradiation which, compared with the measured time of $4 \mathrm{~s}$ (Meyer et al., 2008), gives $t_{d} \approx 10 \mathrm{~s}$. On a $t_{d}$ time scale, we can ignore inertia in (10)-(11).

\subsection{Nondimensional equations in primitive coordinates}

We now transform (10)-(11) to the nondimensional primitive coordinates $u^{\prime}, v^{\prime}$ taking $u=$ $a\left(u^{\prime}+v^{\prime} / 2\right), v=\sqrt{3} a v^{\prime} / 2$, use the nondimensional time scale $t^{\prime}=t / t_{d}$ and ignore inertia, thereby obtaining

$$
\begin{aligned}
& \partial_{t^{\prime}} u^{\prime}=\frac{4 \mu T u^{\prime}}{\lambda+2 \mu}+\frac{\lambda+\mu}{\lambda+2 \mu}\left[\left(H-\frac{D_{1}-D_{2}}{3}\right) u^{\prime}+\left(H+\frac{D_{1}-D_{2}}{3}-2 T\right) v^{\prime}\right], \\
& \partial_{t^{\prime}} v^{\prime}=\frac{2}{3} \frac{\lambda+\mu}{\lambda+2 \mu}\left(D_{1}-D_{2}\right) u^{\prime}+4 T v^{\prime}+\frac{\lambda+\mu}{\lambda+2 \mu}\left(\frac{D_{1}-D_{2}}{3}-H\right) v^{\prime} .
\end{aligned}
$$

\subsection{Periodized discrete elasticity}

Equations (12) - (13) do not allow for the changes of neighbors involved in defect motion. One way to achieve these changes is to update neighbors as a defect moves. Then (12) and (13) would have the same appearance, but the neighbors $n_{i}$ would be given by (5) only at the start. At each time step, we keep track of the position of the different atoms and update the coordinates of the $n_{i}$. This is commonly done in Molecular Dynamics, as computations are actually carried out with only a certain number of neighbors. Convenient as updating is, its computational cost is high and analytical studies thereof are not easy.

In simple geometries, we can avoid updating by introducing a periodic function of differences in the primitive directions that automatically describes link breakup and union associated with defect motion. Besides greatly reducing computational cost, the resulting periodized discrete elasticity models allow analytical studies of defect depinning and motion (Carpio \& Bonilla, 2003; 2005). Another advantage of periodized discrete elasticity is that boundary conditions can be controlled efficiently to avoid spurious numerical reflections at boundaries.

To restore crystal periodicity, we replace the linear operators $T, H, D_{1}$ and $D_{2}$ in (12) and (13) by their periodic versions:

$$
\begin{aligned}
& T_{p} u^{\prime}=g\left(u^{\prime}\left(n_{1}\right)-u^{\prime}(A)\right)+g\left(u^{\prime}\left(n_{2}\right)-u^{\prime}(A)\right)+g\left(u^{\prime}\left(n_{3}\right)-u^{\prime}(A)\right), \\
& H_{p} u^{\prime}=g\left(u^{\prime}\left(n_{6}\right)-u^{\prime}(A)\right)+g\left(u^{\prime}\left(n_{7}\right)-u^{\prime}(A)\right), \\
& D_{1 p} u^{\prime}=g\left(u^{\prime}\left(n_{4}\right)-u^{\prime}(A)\right)+g\left(u^{\prime}\left(n_{9}\right)-u^{\prime}(A)\right), \\
& D_{2 p} u^{\prime}=g\left(u^{\prime}\left(n_{5}\right)-u^{\prime}(A)\right)+g\left(u^{\prime}\left(n_{8}\right)-u^{\prime}(A)\right),
\end{aligned}
$$

where $g$ is a periodic function, with period one, and such that $g(x) \sim x$ as $x \rightarrow 0$. In this work, $g$ is a periodic piecewise linear continuous function:

$$
g(x)= \begin{cases}x, & -\alpha \leq x \leq \alpha, \\ -\frac{2 \alpha}{1-2 \alpha} x+\frac{\alpha}{1-2 \alpha}, & \alpha \leq x \leq 1-\alpha .\end{cases}
$$

The parameter $\alpha$ controls defect stability and mobility under applied stress. It should be sufficiently large for elementary defects (dislocations, vacancies) to be stable at zero applied stress, and sufficiently small for dislocations to move under reasonable applied stress (Carpio \& Bonilla, 2005). We use $\alpha=0.4$ to account for experimentally observed stability 
properties of the defects. For lower values, the stable defect described in section 4 loses the Stone-Wales component. The periodic function $g$ can be replaced by a different type of periodic function to achieve a better fit to available experimental or numerical data.

\section{Stable cores of dislocations and dislocation dipoles in planar graphene}

\subsection{Boundary and initial conditions for a single dislocation}

We solve (12)-(13) with the periodic operators $T_{p}, H_{p}, D_{1 p}$ and $D_{2 p}$, using as initial and boundary conditions the far field of appropriate dislocations which are the stationary solutions of the linear elasticity equations (Landau \& Lifshitz, 1986). Since the latter are a good approximation four spacings away from the core of SW defects in graphene, and our model equations seamlessly reduce to linear elasticity in the far field, we use a relatively small lattice with $19 \times 19$ spacings $(2 \times 18 \times 18$ carbon atoms $)$ in our numerical simulations (Carpio \& Bonilla, 2008). Consider first the case of a single edge dislocation with Burgers vector $(a, 0)$ and displacement vector $\mathbf{u}=(u(x, y), v(x, y))$

$$
\begin{aligned}
& u=\frac{a}{2 \pi}\left[\tan ^{-1}\left(\frac{y}{x}\right)+\frac{x y}{2(1-v)\left(x^{2}+y^{2}\right)}\right], \\
& v=\frac{a}{2 \pi}\left[-\frac{1-2 v}{4(1-v)} \ln \left(\frac{x^{2}+y^{2}}{a^{2}}\right)+\frac{y^{2}}{2(1-v)\left(x^{2}+y^{2}\right)}\right],
\end{aligned}
$$

where $v=\lambda /[2(\lambda+\mu)]$ is dimensionless; cf. page 114 of (Landau \& Lifshitz, 1986). (16) has a singularity $\propto\left(x^{2}+y^{2}\right)^{-1 / 2}$ at the origin of coordinates and it satisfies $\int_{\mathcal{C}}(d \mathbf{x} \cdot \nabla) \mathbf{u}=-(a, 0)$, for any closed curve $\mathcal{C}$ encircling the origin. Using (16), we write $\mathbf{u}=(u, v)$ in primitive coordinates, $U^{\prime}(l, m)=\left[u\left(x-x_{0}, y-y_{0}\right)-v\left(x-x_{0}, y-y_{0}\right) / \sqrt{3}\right] / a, V^{\prime}(l, m)=2 v\left(x-x_{0}, y-\right.$ $\left.y_{0}\right) /(a \sqrt{3})$, where $x=\left(x^{\prime}+y^{\prime} / 2\right) a, y=\sqrt{3} a y^{\prime} / 2, x^{\prime}=l, y^{\prime}=m$ (integers) and $\left(x_{0}, y_{0}\right) \neq$ $(0,0)$ to avoid that the singularity in (16) be placed at a lattice point. To find defects, we solve the periodized versions of the discrete elasticity equations (12)-(13) with the initial and boundary conditions:

$$
\mathbf{u}^{\prime}(l, m ; 0)=\mathbf{U}^{\prime}(l, m), \quad \text { and } \quad \mathbf{u}^{\prime}(l, m ; t)=\mathbf{U}^{\prime}(l, m)+F(m, 0) \text { at lattice boundaries. }
$$

Here $F$ is a dimensionless applied shear stress. For $|F|<F_{c}$ (Peierls stress), the solution of the periodized version of (12)-(13) relaxes to a stable dislocation $\left(u^{\prime}(l, m), v^{\prime}(l, m)\right)$ with appropriate far field, which is (16) if $F=0$.

Numerical simulations give us the location of carbon atoms at each time $t$. We represent atoms by spheres of arbitrary size. As a guide to the eye and to visualize defects more easily, we have attached fictitious bonds to these spheres (Carpio \& Bonilla, 2008; Carpio et al., 2008). Depending on the location of the singularity $\left(x_{0}, y_{0}\right)$, there are two possible configurations corresponding to the same edge dislocation in the continuum limit. If $\left(x_{0}, y_{0}\right)$ is placed between two atoms that form any non-vertical side of a given hexagon, the core of the deformed lattice $\left(l+u^{\prime}(l, m), m+v^{\prime}(l, m)\right)$ is a 5-7 (pentagon-heptagon) defect. If $\left(x_{0}, y_{0}\right)$ is placed in any other location different from a lattice point, the core of the singularity forms an octagon having one atom with a dangling bond (Carpio \& Bonilla, 2008; Carpio et al., 2008). Stable 5-7 defects are commonly observed in experiments (Gómez-Navarro et al., 2010; Meyer et al., 2008; 2009), whereas adsorbed atoms (not considered in our model) may attach to a dangling bond thereby destroying the octagon configuration. 


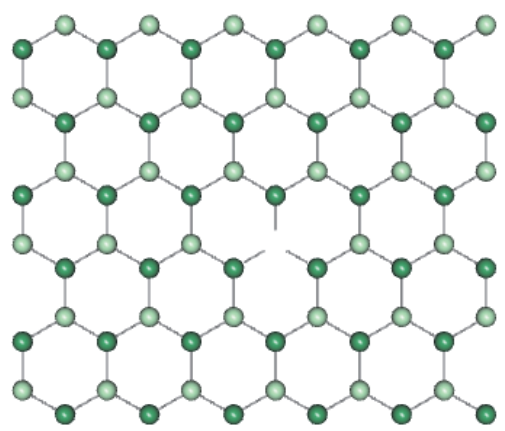

a)

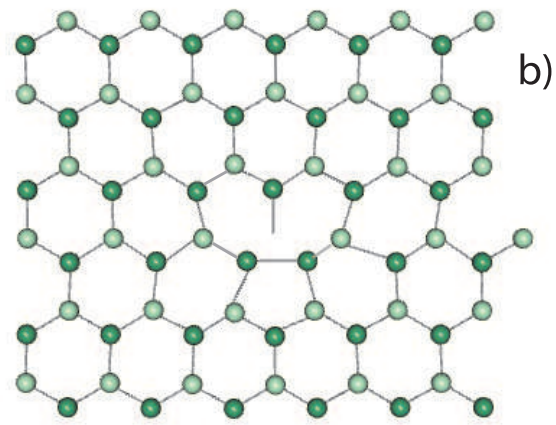

Fig. 2. (a) Symmetric vacancy. (b) Asymmetric vacancy (nonagon-pentagon defect).

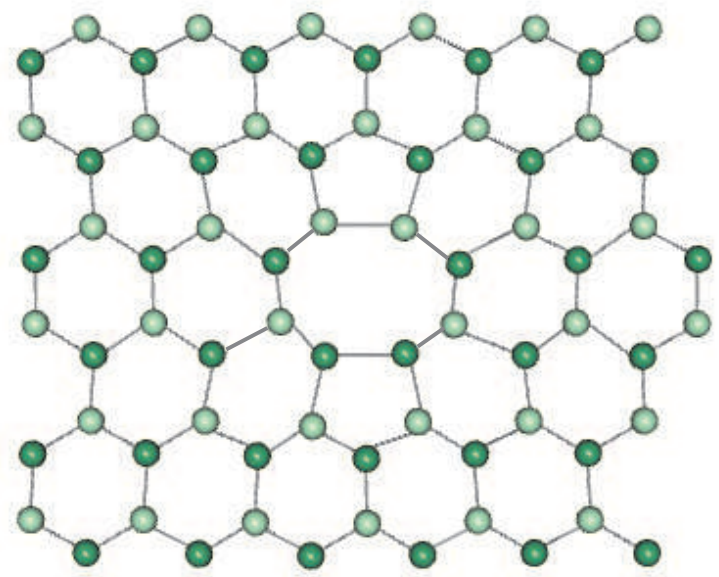

Fig. 3. Pentagon-octagon-pentagon divacancy.

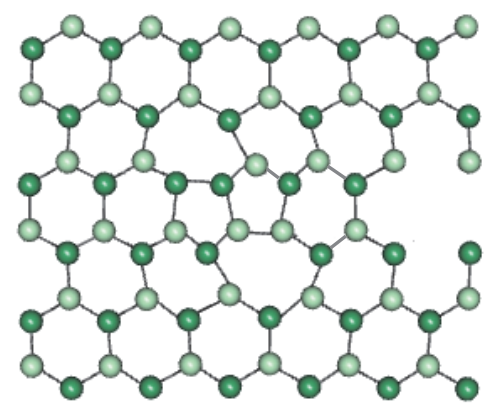

a)

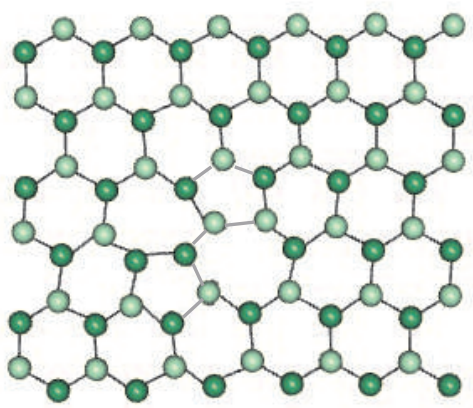

b)

Fig. 4. (a) Stable 7-5-5-7 defect. (b) Unstable 5-7-7-5 Stone Wales defect. 


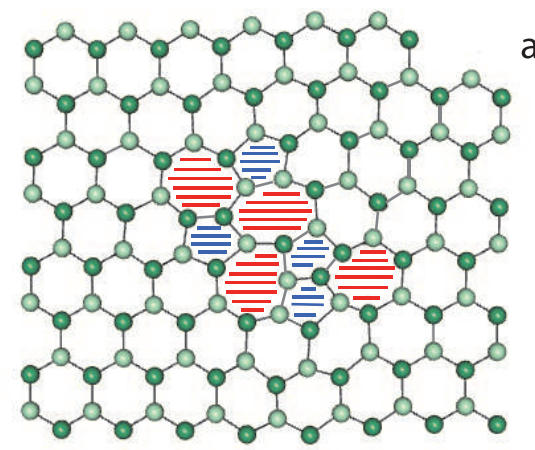

a)

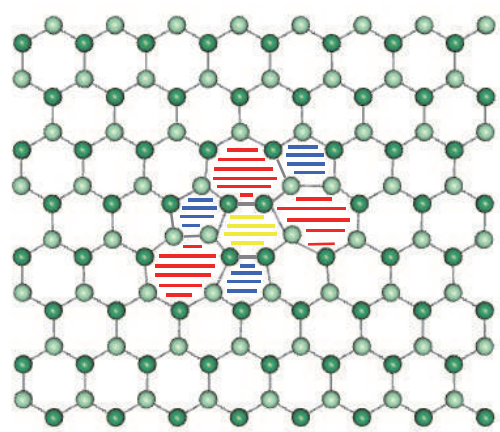

b)

Fig. 5. Defects corresponding to two dislocation dipoles: (a) Stable configuration comprising a pair of 5-7-7-5 and 7-5-5-7 defects. (b) Metastable defect comprising three heptagons, three pentagons and one hexagon.

\subsection{Dislocation dipoles}

A dislocation dipole is formed by two dislocations with opposite Burgers vectors, $\pm \mathbf{a}$. Depending on how we place the origin of coordinates, different dipole configurations result. Let $\mathbf{U}(x, y)$ be the displacement vector (16) of a single dislocation. We find the dipole cores by selecting as zero stress initial and boundary conditions $\mathbf{U}\left(x-x_{0}^{+}, y-y_{0}^{+}\right)-\mathbf{U}\left(x-x_{0}^{-}, y-y_{0}^{-}\right)$, with different $\left(x_{0}^{ \pm}, y_{0}^{ \pm}\right)$. Let $a, L=a / \sqrt{3}, H=3 L / 2$ and $h=L / 2$ be the lattice constant, the hexagon side, the vertical distance between two nearest neighbor atoms belonging to the same sublattice separated by one primitive vector $\mathbf{b}$ or $\mathbf{c}$, and the vertical distance between nearest neighbor atoms having different ordinate, respectively. We get:

- Vacancies: $x_{0}^{+}=-0.25 a, y_{0}^{+}=-0.8 h+H / 2$ and $x_{0}^{-}=-0.25 a, y_{0}^{-}=-0.8 h$. This initial configuration is the asymmetric vacancy (9-5 defect) of Fig. 2(b), which evolves to the symmetric vacancy of Fig. 2(a) under overdamped dynamics.

- Stable divacancy: $x_{0}^{+}=-0.25 a, y_{0}^{+}=-0.8 h+H$ and $x_{0}^{-}=-0.25 a, y_{0}^{-}=-0.8 h$. Fig. 3 .

- Stable 7-5-5-7 defect: $x_{0}^{+}=-0.25 a+a, y_{0}^{+}=-0.8 h$ and $x_{0}^{-}=-0.25 a, y_{0}^{-}=-0.8 h+H$. Fig. 4(a).

- Unstable Stone-Wales 5-7-7-5 defect: $x_{0}^{+}=-0.25 a+a, y_{0}^{+}=-0.8 h$ and $x_{0}^{-}=-0.25 a$, $y_{0}^{-}=-0.8 h$. Fig. 4(b). For $F=0$, this initial configuration corresponds to two dislocations with opposite Burgers vectors that share the same glide line, and it evolves to the undisturbed lattice when the dislocations move towards each other and annihilate.

\subsection{Dislocation dipole pairs}

We now study the evolution of configurations comprising two dislocation dipoles each. Fig. 5(a) depicts a stable defect consisting of a 5-7-7-5 SW defect adjacent to a rotated 7-5-5-7 defect:

$$
\begin{aligned}
& \mathbf{U}\left(x-x_{0}^{+}, y-y_{0}^{+}\right)-\mathbf{U}\left(x-x_{0}^{-}, y-y_{0}^{-}\right)+\tilde{\mathbf{U}}\left(\tilde{x}-\tilde{x}_{0}^{+}, \tilde{y}-\tilde{y}_{0}^{+}\right)-\tilde{\mathbf{U}}\left(\tilde{x}-\tilde{x}_{0}^{-}, \tilde{y}-\tilde{y}_{0}^{-}\right), \\
& x_{0}^{+}=-0.3 a, y_{0}^{+}=-0.7 h+2 H, \quad x_{0}^{-}=-0.3 a-a, y_{0}^{-}=-0.7 h+2 H, \\
& \tilde{x}_{0}^{+}=0.3 a+a, \tilde{y}_{0}^{+}=0.3 h-H, \quad \tilde{x}_{0}^{-}=0.15 a, \tilde{y}_{0}^{+}=0.5 h .
\end{aligned}
$$


Here $\mathbf{U}(x, y)$ is the edge dislocation (16) with origin of coordinates at a central atom of type $A$ in Figure 1 and Burgers vector a (in units of the lattice constant $a$ ). $\tilde{\mathbf{U}}(\tilde{x}, \tilde{y})$ is an edge dislocation with Burgers vector $\mathbf{b}$. To obtain $\tilde{\mathbf{U}}(\tilde{x}, \tilde{y})$ in (18), we consider the axes $(\tilde{x}, \tilde{y})$ rotated a $\pi / 3$ angle from the axes $(x, y)$. Next we form a 7-5-5-7 defect by combining a positive dislocation with Burgers vector $(a, 0)$ centered at $\left(\tilde{x}_{0}^{+}, \tilde{y}_{0}^{+}\right)$and a negative dislocation with Burgers vector $(-a, 0)$ centered at $\left(\tilde{x}_{0}^{-}, \tilde{y}_{0}^{-}\right)$. Then the result is rewritten in the original coordinates $(x, y)$. This is the same defect as reported in Figures 3(h) and (i) of Meyer et al's experiments (Meyer et al., 2008) and it remains stable under overdamped dynamics. The 7-5-5-7 defect is stable (Carpio \& Bonilla, 2008; Carpio et al., 2008), and this apparently stabilizes our pair of dislocation dipoles for the selected initial configuration. Other nearby configurations evolve to two octagons corresponding to a dipole comprising two edge dislocations with opposite Burgers vectors. As explained before, adsorbed atoms may be attached to the dangling bonds thereby eliminating these configurations and restoring the undisturbed hexagonal lattice.

Let us consider now two dislocation dipoles all whose component dislocations have Burgers vectors directed along the $x$ axis according to the initial and boundary condition depicted in Figure 5(b) (a defect comprising three pentagons and three heptagons):

$$
\begin{aligned}
& \mathbf{U}\left(x-x_{0}^{+}, y-y_{0}^{+}\right)-\mathbf{U}\left(x-x_{0}^{-}, y-y_{0}^{-}\right)+\mathbf{U}\left(x-\hat{x}_{0}^{+}, y-\hat{y}_{0}^{+}\right)-\mathbf{U}\left(x-\hat{x}_{0}^{-}, y-\hat{y}_{0}^{-}\right), \\
& x_{0}^{+}=-0.3 a+a, y_{0}^{+}=-0.7 h, \quad x_{0}^{-}=-0.3 a, y_{0}^{-}=-0.7 h, \\
& \hat{x}_{0}^{+}=-0.3 a-a, \hat{y}_{0}^{+}=-0.7 h-H, \quad \hat{x}_{0}^{-}=-0.3 a, \hat{y}_{0}^{+}=-0.7 h-H .
\end{aligned}
$$

Starting from a negative dislocation centered at $\left(x_{0}^{-}, y_{0}^{-}\right)=(-0.3 a,-0.7 h)$, the first dipole adds a positive dislocation shifted one lattice constant to the right. The second dipole consists of a negative dislocation shifted vertically downwards a distance $H=3 L / 2=\sqrt{3} a / 2(1.5$ times the hexagon side, or $\sqrt{3} / 2$ times the lattice constant) from $\left(x_{0}^{-}, y_{0}^{-}\right)$and a positive dislocation which shifts horizontally to the left the previous one a distance equal to one lattice constant. Under overdamped dynamics, this defect disappears as the positive and negative dislocations comprising each dipole glide towards each other.

\subsection{Comparison with results of experiments}

Carbon atoms and defects in graphene sheets are visualized by operating at low voltage $(\leq 80$ $\mathrm{kV}$, to avoid irradiation damage to the sample) a transmission electron aberration-corrected microscope (TEAM) with appropriate optics (Meyer et al., 2008). This microscope is capable of sub-Ångstrom resolution even at $80 \mathrm{kV}$ and can produce real time images of carbon atoms on a scale of seconds: each frame averages 1 s of exposure and the frames themselves are $4 \mathrm{~s}$ apart (Girit et al., 2009; Meyer et al., 2008). The images obtained in experiments can be used to determine the time evolution of defects in graphene created by irradiation or sample treatment (Girit et al., 2009; Gómez-Navarro et al., 2010; Meyer et al., 2008; 2009).

As explained before, stable pentagon-heptagon defects but not isolated octagons with a dangling bond are observed in experiments (Gómez-Navarro et al., 2010; Meyer et al., 2008; 2009). The octagon configuration is quite reactive and may be destroyed by attaching an adsorbed carbon atom, which is not contemplated in our model. Our theory indicates that symmetric vacancies are stable and asymmetric ones are unstable. In experiments, both symmetric and asymmetric vacancies are observed in unstressed graphene (Meyer et al., 2008), whereas in single layers of hexagonal Boron Nitride (hBN) only symmetric vacancies 
are observed (Meyer et al., 2009), as predicted by our theory. See Carpio \& Bonilla (2008) for a possible mechanism to produce stable asymmetric vacancies in graphene. Stable 5-8-5 divacancies are also observed (Coleman et al., 2008). The annihilation of the 5-7-7-5 SW defect in 4(b) (the heptagons share one side) $4 \mathrm{~s}$ after its creation is seen in Figures 3(c) and (d) of the paper by Meyer et al. (2008). Our model predicts that SW under sufficient strain split in their two 5-7 pairs that move apart (cf Fig 6 in Carpio \& Bonilla (2008)), which has been observed very recently; cf Fig. 4(a) and (b) in Gómez-Navarro et al. (2010). Of the two pairs of dislocation dipoles, the defect comprising four pentagons and four heptagons was observed to be stable in Figures 3(h) and (i) in Meyer et al. (2008), whereas the defect comprising three pentagons, three heptagons and one hexagon disappeared after a short time (Meyer et al., 2008), as predicted by the numerical simulations of our model equations.

\section{Ripples and defects in a three dimensional suspended graphene sheet}

The first observations of suspended graphene sheets showed evidence of ripples: ondulations of the sheet with characteristic amplitudes and wave lengths (Meyer et al., 2007). Monte Carlo calculations also showed ripple formation (Fasolino et al., 2007) and explored the possibility that the ripples are due to thermal fluctuations; see also Abedpour et al. (2007). According to Thompson-Flagg et al. (2009), thermal fluctuations would produce much smaller ripples than observed in experiments and therefore this explanation does not seem likely. Thompson-Flagg et al. (2009) explain ripples as a consequence of adsorbed $\mathrm{OH}$ molecules on random sites. More recent experiments on graphene sheets suspended on substrate trenches have shown that ripples can be thermally induced and controlled by thermal cycling in a clean atmosphere (Bao et al., 2009) which seems to preclude the explanation based on adsorbed $\mathrm{OH}$ molecules (Thompson-Flagg et al., 2009). Bao et al. (2009) use the difference in the thermal expansion coefficients of the graphene sheet (negative coefficient) and its pinning substrate (positive coefficient) to vary the tension in the sheet which, in turn, governs the ripples (Bao et al., 2009; Bunch et al., 2007).

In this section, we present a theory of ripples and defects in suspended graphene sheets based on periodized discrete elasticity (Bonilla \& Carpio, 2011). A suspended graphene sheet may bend upwards or downwards and therefore it may be described by the free energy of the membrane (1) with $w \neq 0$ in the continuum limit. Carbon atoms in the graphene sheet have $\sigma$ bond orbitals constructed from $s p^{2}$ hybrid states oriented in the direction of the bond that accommodate three electrons per atom. The remaining electrons go to $p$ states oriented perpendicularly to the sheet. These orbitals bind covalently with neighboring atoms and form a narrow $\pi$ band that is half-filled. The presence of bending and ripples in graphene modifies its electronic structure (Castro Neto et al., 2009). Out-of-plane convex or concave deformations of the sheet have in principle equal probability and transitions between these deformations are associated with the bending energy of the sheet. A simple way to model this situation to consider that out-of-plane deformations are described by the values of an Ising spin associated to each carbon atom. Then the vertical coordinate of each atom in the graphene sheet is coupled to an Ising spin $\sigma= \pm 1$ in contact with a bath at temperature $T$ and that the spin system has an energy

$$
F_{s}=-f \int \sigma(x, y) w(x, y) d x d y,
$$


in the continuum limit. $f$ has units of force per unit area. The spins flip stochastically according to Glauber dynamics at temperature $\theta$ (measured in units of energy). Thus at any time $t$, the system may experience a transition from $(\boldsymbol{u}, \boldsymbol{v}, \boldsymbol{w}, \boldsymbol{\sigma})$ to $\left(\boldsymbol{u}, \boldsymbol{v}, \boldsymbol{w}, R_{(x, y)} \boldsymbol{\sigma}\right)$ at a rate given by

$$
W_{(x, y)}(\boldsymbol{\sigma} \mid \boldsymbol{u}, \boldsymbol{v}, \boldsymbol{w})=\frac{\zeta}{2}[1-\beta(x, y) \sigma(x, y)], \quad \beta(x, y)=\tanh \left(\frac{f a^{2} w(x, y)}{\theta}\right),
$$

(Glauber, 1963), where $R_{(x, y)} \boldsymbol{\sigma}$ is the configuration obtained from $\sigma=\{\sigma(x, y)\}$ (for all points $(x, y)$ on the hexagonal lattice) by flipping the spin at the lattice point $(x, y)$. The parameter $\zeta$ gives the characteristic attempt rate for the transitions in the Ising system. Since the bending energy of the graphene sheet is $\kappa$, the attempt rate should be proportional to an Arrhenius factor:

$$
\zeta=\zeta_{0} \exp \left(-\frac{\kappa}{\theta}\right)
$$

where $\zeta_{0}$ is a constant.

The total free energy,

$$
F=F_{g}+F_{s}=\int\left[\frac{\kappa}{2}\left(\nabla^{2} w\right)^{2}+\frac{\lambda}{2} u_{i i}^{2}+\mu u_{i k}^{2}-f \sigma w\right] d x d y
$$

is obtained from (1) and (22) and it provides the equations of motion (Bonilla \& Carpio, 2011):

$$
\begin{aligned}
\rho_{2} \partial_{t}^{2} u & =\lambda \partial_{x}\left(\partial_{x} u+\partial_{y} v+\frac{\left(\partial_{x} w\right)^{2}+\left(\partial_{y} w\right)^{2}}{2}\right)+\mu \partial_{x}\left[2 \partial_{x} u+\left(\partial_{x} w\right)^{2}\right] \\
& +\mu \partial_{y}\left(\partial_{y} u+\partial_{x} v+\partial_{x} w \partial_{y} w\right) \\
\rho_{2} \partial_{t}^{2} v & =\lambda \partial_{y}\left(\partial_{x} u+\partial_{y} v+\frac{\left(\partial_{x} w\right)^{2}+\left(\partial_{y} w\right)^{2}}{2}\right)+\mu \partial_{y}\left[2 \partial_{y} v+\left(\partial_{y} w\right)^{2}\right] \\
& +\mu \partial_{x}\left(\partial_{y} u+\partial_{x} v+\partial_{x} w \partial_{y} w\right)
\end{aligned}
$$

instead of (3)-(4) and

$$
\begin{aligned}
\rho_{2} \partial_{t}^{2} w & =-\kappa\left(\nabla^{2}\right)^{2} w+\lambda \partial_{x}\left[\left(\partial_{x} u+\partial_{y} v+\frac{\left(\partial_{x} w\right)^{2}+\left(\partial_{y} w\right)^{2}}{2}\right) \partial_{x} w\right] \\
& +\lambda \partial_{y}\left[\left(\partial_{x} u+\partial_{y} v+\frac{\left(\partial_{x} w\right)^{2}+\left(\partial_{y} w\right)^{2}}{2}\right) \partial_{y} w\right] \\
& +\mu \partial_{x}\left\{2 \partial_{x} u \partial_{x} w+\left(\partial_{y} u+\partial_{x} v\right) \partial_{y} w+\left[\left(\partial_{x} w\right)^{2}+\left(\partial_{y} w\right)^{2}\right] \partial_{x} w\right\} \\
& +\mu \partial_{y}\left\{\left(\partial_{y} u+\partial_{x} v\right) \partial_{x} w+2 \partial_{y} v \partial_{y} w+\left[\left(\partial_{x} w\right)^{2}+\left(\partial_{y} w\right)^{2}\right] \partial_{y} w\right\}+f \sigma,
\end{aligned}
$$

for $w$. In this section, we omit the phenomenological friction (with coefficient $\gamma$ ) in the equations of motion because the stochastic Glauber dynamics already provides an effective friction for the spins which try to reach equilibrium at the bath temperature $\theta$. The periodized discrete elasticity corresponding to these equations is derived in the same fashion as for planar graphene in Section 3, except that we need to add the new difference operators to those in 
(6)-(9):

$$
\begin{aligned}
& \Delta_{h} u=u\left(n_{7}\right)-u(A) \sim\left(\partial_{x} u\right) a, \\
& \Delta_{v} u=u\left(n_{3}\right)-u(A) \sim\left(\partial_{y} u\right) \frac{a}{\sqrt{3}}, \\
& B w=\left[\operatorname{Tw}\left(n_{1}\right)-\operatorname{Tw}(A)\right]+\left[\operatorname{Tw}\left(n_{2}\right)-\operatorname{Tw}(A)\right]+\left[\operatorname{Tw}\left(n_{3}\right)-\operatorname{Tw}(A)\right] \sim \frac{a^{4}}{16}\left(\nabla^{2}\right)^{2} w,
\end{aligned}
$$

as $a \rightarrow 0$. The discrete elasticity equations are now (Bonilla \& Carpio, 2011):

$$
\begin{aligned}
\rho_{2} a^{2} \partial_{t}^{2} u & =4 \mu T u+(\lambda+\mu) H u+\frac{\lambda+\mu}{\sqrt{3}}\left(D_{1}-D_{2}\right) v+\frac{4 \mu}{a} \Delta_{h} w T w \\
& +\frac{\lambda+\mu}{a}\left[\Delta_{h} w H w+\Delta_{v} w\left(D_{1}-D_{2}\right) w\right] \\
\rho_{2} a^{2} \partial_{t}^{2} v & =4(\lambda+2 \mu) T v-(\lambda+\mu) H v+\frac{\lambda+\mu}{\sqrt{3}}\left(D_{1}-D_{2}\right) u+\frac{4 \sqrt{3}}{a}(\lambda+2 \mu) \Delta_{v} w T w \\
& +\frac{\lambda+\mu}{a \sqrt{3}}\left[\Delta_{h} w\left(D_{1}-D_{2}\right) w-3 \Delta_{v} w H w\right], \\
\rho_{2} a^{2} \partial_{t}^{2} w & =\frac{\lambda+2 \mu}{a}\left\{\left[H u+\frac{\Delta_{h} w}{a} H w+\frac{2 \Delta_{v} w}{a}\left(D_{1}-D_{2}\right) w\right] \Delta_{h} w+[\sqrt{3}(4 T-H) v\right. \\
& \left.\left.+\frac{3 \Delta_{v} w}{a}(4 T-H) w\right] \Delta_{v} w\right\}+\frac{\lambda+\mu}{a}\left(D_{1}-D_{2}\right) u \Delta_{v} w+\frac{\lambda \Delta_{h} w}{\sqrt{3} a}\left(D_{1}-D_{2}\right) v \\
& +\frac{\mu \Delta_{v} w}{a}\left[\sqrt{3}(4 T-H) u+\left(D_{1}-D_{2}\right) v+\sqrt{3} H v\right]+\frac{2 \Delta_{h} u}{a}(2 \lambda T+\mu H) w \\
& +\frac{2 \sqrt{3} \Delta_{v} v}{a}[2 \lambda T w+\mu(4 T-H) w]+\frac{2 \mu}{a}\left(\Delta_{v} u+\frac{\Delta_{h} v}{\sqrt{3}}\right)\left(D_{1}-D_{2}\right) w \\
& +\frac{\left(\Delta_{h} w\right)^{2}+\left(\Delta_{v} w\right)^{2}}{a^{2}}(2 \lambda T+\mu H) w+\frac{4 \mu}{a^{2}} T w(4 T-H) w-\frac{16 \kappa}{a^{2}} B w+f a^{2} \sigma .
\end{aligned}
$$

We have periodized these equations after writing them in primitive coordinates, adopted the nondimensional time scale $t^{\prime}=t /[t]$ as in (12)-(13) and solved them numerically. In our simulations, we have used the numerical values of the isothermal elastic moduli at $300 \mathrm{~K}$ provided by Zakharchenko et al. (2009), $\lambda=2.57 \mathrm{eV} / \AA^{2}, \mu=9.95 \mathrm{eV} / \AA^{2}$, which agree well with experiments (Lee et al., 2008) and with the values for graphite at the basal plane (Blakslee et al., 1970). The bending coefficient at $300 \mathrm{~K}$ is $\kappa=1.08 \mathrm{eV}$ (Zakharchenko et al., 2009). The time constant is $[t]=a \sqrt{\rho_{2} /(\lambda+2 \mu)}=1.1033 \times 10^{-14}$ s because $a=2.461 \AA$ and we get $\rho_{2}=2160 \times 3.35 \times 10^{-10}=7.236 \times 10^{-7} \mathrm{~kg} / \mathrm{m}^{2}$ from the three-dimensional mass density of graphite (the distance between graphene planes in graphite is $3.35 \AA$ ). We use $f=0.378 \mathrm{meV}$ and $1 / \zeta=10 \mathrm{~s}$ (Bonilla \& Carpio, 2011). $1 / \zeta$ is the characteristic time it takes a spin to flip, i.e., the time it takes a portion of the graphene sheet to change its concavity. We equal this time to $t_{d}=10 \mathrm{~s}$, the characteristic time for defect motion mentioned in Section 2; see also Carpio \& Bonilla (2008). The ratio between $[t]$ and the flipping time is very small $\delta=\zeta[t]=1.1033 \times 10^{-15}$. In our simulations, we have used a larger value of $\delta$ which shortens 


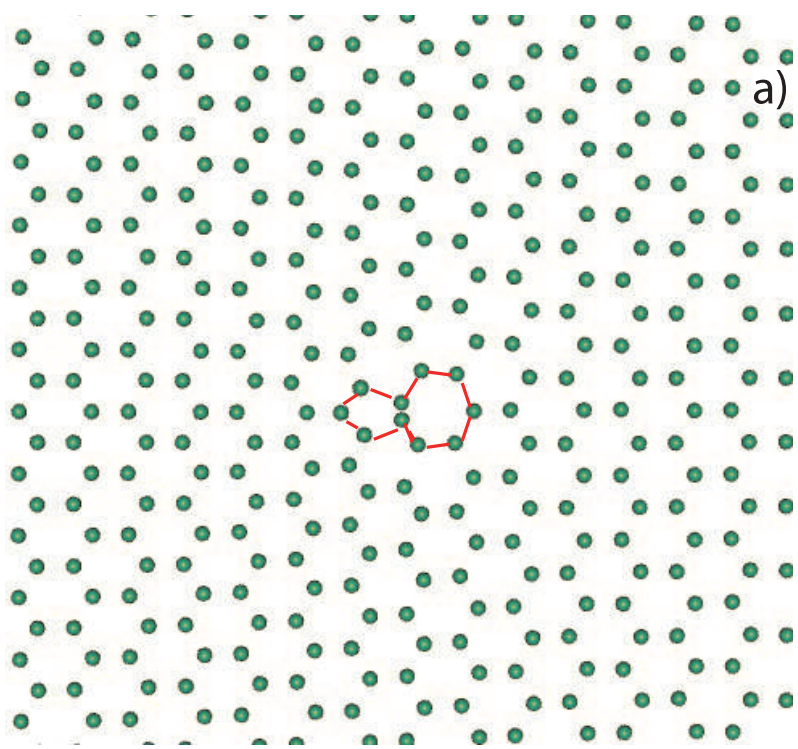

b)
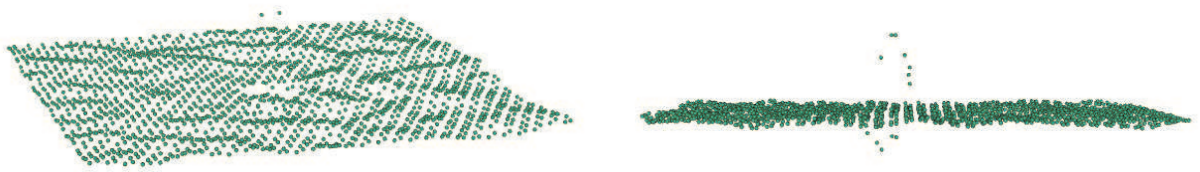

Fig. 6. Suspended graphene sheet containing one pentagon-heptagon defect. (a) View from above the sheet; (b) side view showing that the atoms forming the pentagon move above the plane of the sheet; (c) farther side view showing that there are displaced atoms above the sheet (pentagon) and below it (heptagon). (b) and (c) show that the height of the ripples is smaller than the vertical displacement of the atoms near the defect.

the relaxation time it takes the graphene sheet to reach a stable configuration without changing the latter.

We solve the equations of motion with the same boundary and initial conditions for the in-plane displacement vector $(u, v)$ corresponding to a single pentagon-heptagon defect as explained in Section 3. We also impose that the vertical displacement $w$ vanishes at the border atoms and for fictitious border atoms whose displacements are required when solving the equations of motion. Figure 6 shows a suspended graphene sheet with a pentagon-heptagon defect. At $300 \mathrm{~K}$, the graphene sheet is below its critical temperature and therefore a rippled state is stable (Bonilla \& Carpio, 2011). Thus ripples similar to those observed in experiments (Meyer et al., 2007) coexist with a local curvature near the defect as shown in Figure 6. 


\section{Conclusions}

We have presented a theory of defects in suspended graphene sheets based on periodized discrete elasticity. The equations of linear elasticity are discretized on a hexagonal lattice, written in primitive coordinates and finite differences along primitive directions are replaced for periodic functions thereof. The latter allow for gliding of dislocations along primitive directions because spatial periodicity along them is restored in the equations of motion. Ignoring vertical displacement, we solve these equations numerically assuming that the initial and boundary conditions are given by the known linear elasticity expressions corresponding to one or several dislocations. If the equations of motion include an appropriate damping coefficient, we obtain the stable cores of the dislocations which are defects in the hexagonal lattice. The damping coefficient is fitted to experiments. The stable cores predicted by our theory have been observed in experiments.

We have also proposed a mechanism to explain ripples and curvature in suspended graphene sheets. We assume that the local trend of the sheet to bend upward or downward is represented by an Ising spin coupled to the carbon atoms. These spins are in contact with a thermal bath at the lattice temperature and flip stochastically according to Glauber dynamics. Our simulations show the appearance of ripples in the graphene sheet and the local curvature that appears near a defect, for instance near a pentagon-heptagon defect which is the core of an edge dislocation.

This work has been financed by the Spanish Ministry of Science and Innovation (MICINN) under grants FIS2008-04921-C02-01 (LLB), FIS2008-04921-C02-02 and UCM/BSCH CM 910143 $(\mathrm{AC})$.

\section{References}

Abedpour, N.; Neek-Amal, M.; Asgari, R.; Shahbazi, F.; Nafari, N. \& Rahimi Tabar, M. R. (2007). Roughness of undoped graphene and its short-range induced gauge field, Physical Review B Vol. 76: 195407.

Bao, W.; Miao, F.; Chen, Z.; Zhang, H.; Jang, W.; Dames, C. \& Lau, C.-N. (2009). Controlled Ripple Texturing of Suspended Graphene and Ultrathin Graphite Membranes, Nature Nanotechnology Vol. 4: 562-566.

Blakslee, O. L.; Proctor, D. G.; Seldin, E. J.; Spence, G. B. \& Weng, T. (1970). Elastic Constants of Compression-Annealed Pyrolytic Graphite, Journal of Applied Physics Vol. 41: 3373-3382.

Bonilla, L.L. \& Carpio, A. (2011). Theory of ripples in graphene, Preprint.

Bunch, J.S.; van der Zande, A. M.; Verbridge, S.S.; Tanenbaum, D.M.; Parpia, J.M.; Craighead, H.G. \& McEuen, P.L. (2007). Electromechanical Resonators from Graphene Sheets, Science Vol. 315: 490-493.

Carpio, A. \& Bonilla, L.L. (2003). Edge dislocations in crystal structures considered as traveling waves of discrete models, Physical Review Letters Vol. 90: 135502.

Carpio, A. \& Bonilla, L.L. (2005). Discrete models of dislocations and their motion in cubic crystals, Physical Review B Vol. 71: 134105.

Carpio, A. \& Bonilla, L.L. (2008). Periodized discrete elasticity models for defects in graphene, Physical Review B Vol. 78: 085406.

Carpio, A.; Bonilla, L.L.; Juan, F. de \& Vozmediano, M.A.H. (2008). Dislocations in graphene, New Journal of Physics Vol. 10: 053021. 
Castro Neto, A. H.; Guinea, F.; Peres, N. M. R.; Novoselov, K. S. \& Geim, A. K. (2009). The electronic properties of graphene, Reviews of Modern Physics Vol. 81: 109-162.

Coleman, V. A.; Knut, R.; Karis, O.; Grennberg, H.; Jansson, U.; Quinlan, R.; Holloway, B. C.; Sanyal, B. \& Eriksson, O. (2008). Defect formation in graphene nanosheets by acid treatment: an x-ray absorption spectroscopy and density functional theory study, Journal of Physics D: Applied Physics Vol. 41: 062001.

Fasolino, A.; Los, J.H. \& Katsnelson, M.I. (2007). Intrinsic ripples in graphene,Nature Materials Vol. 6: 858-861.

Geim, A.K. \& Novoselov, K.S. (2007). The rise of graphene, Nature Materials Vol. 6: 183-191.

Geim, A.K. (2009). Graphene: Status and Prospects, Science Vol. 324: 1530-1534.

Girit, C.O., Meyer, J.C., Erni, K., Rossell, M. D., Kisielowski, C., Yang, L., Park, C.-H., Crommie, M. F., Cohen, M. L., Louie, S. G., Zettl, A. (2009). Graphene at the Edge: Stability and Dynamics Science Vol. 323: 1705-1708.

Glauber, R. J. (1963). Time-dependent statistics of the Ising model, Journal of Mathematical Physics Vol. 4: 294-307.

Gómez-Navarro, C., Meyer, J.C., Sundaram, R. S., Chuvilin, A., Kurasch, S., Burghard, M., Kern, K. \& Kaiser, U. (2010). Atomic Structure of Reduced Graphene Oxide, Nano Letters Vol. 10: 1144-1148.

Guinea, F.; Katsnelson, M.I. \& Geim, A.K. (2009). Energy gaps and a zero-field quantum Hall effect in graphene by strain engineering, Nature Physics Vol. 6: 33-33.

Landau, L.D. \& Lifshitz, E.M. (1986). Theory of elasticity, 3rd ed., Pergamon Press, Oxford.

Lee, C.; Wei, X.; Kysar, J.W. \& J. Hone (2008). Measurement of the Elastic Properties and Intrinsic Strength of Monolayer Graphene, Science Vol. 321: 385-388.

Meyer, J.C.; Geim, A.K.; Katsnelson, M.I.; Novoselov, K.S.; Booth, T.J. \& Roth, S. (2007). The structure of suspended graphene sheets, Nature Vol. 446: 60-63.

Meyer, J. C.; Kisielowski, C.; Erni, R.; Rossell, M.D.; Crommie M.F. \& Zettl, A. (2008). Direct imaging of lattice atoms and topological defects in graphene membranes, Nano Letters Vol. 8(No. 11): 3582-3586.

Meyer, J.C., Chuvilin, A., Algara-Siller, G., Biskupek, J. \& Kaiser, U. (2009). Selective Sputtering and Atomic Resolution Imaging of Atomically Thin Boron Nitride Membranes, Nano Letters Vol. 9: 2683-2689.

Nelson, D. R. (2002). Defects and Geometry in Condensed Matter Physics, Cambridge U.P., Cambridge.

Novoselov, K.S.; Geim, A. K.; Morozov, S. V.; Jiang, D.; Zhang, Y.; Dubonos, S. V.; Grigorieva, I. V. \& Firsov, A. A.(2004). Electric field effect in atomically thin carbon films, Science Vol. 306: 666-669.

Novoselov, K.S.; Jiang,D.; Schedin, F.; Booth, T.J.; Khotkevich, V. V.; Morozov, S. V. \& Geim, A. K. (2005). Two-dimensional atomic crystals, Proceedings of the National Academy of Sciences USA Vol.102: 10451-10453.

Segall, M.D.; Lindan, P. J. D.; Probert, M.J.; Pickard, C.J.; Hasnip, P. J.; Clark, S.J. \& Payne, M. C. (2002). First-principles simulation: ideas, illustrations and the CASTEP code, Journal of Physics: Condensed Matter Vol. 14: 2717-2744.

Thompson-Flagg, R. C.; Moura, M. J. B. \& Marder, M. (2009). Rippling of graphene, Europhys. Letters Vol. 85: 46002.

Vozmediano, M.A.H.; Katsnelson, M.I. \& Guinea, F. (2010). Gauge fields in graphene, Physics Reports Vol. 496: 109-148. 
Wang, X.; Tabakman, S. M. \& Dai, H. (2008). Atomic Layer Deposition of Metal Oxides on Pristine and Functionalized Graphene, Journal of the American Chemical Society Vol. 130: 8152-8153.

Zakharchenko, K. V.; Katsnelson, M. I. \& Fasolino, A. (2009). Finite Temperature Lattice Properties of Graphene beyond the Quasiharmonic Approximation, Physical Review Letters Vol. 102: 046808. 


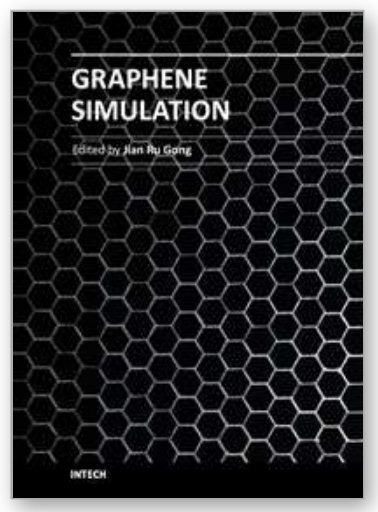

\author{
Graphene Simulation \\ Edited by Prof. Jian Gong
}

ISBN 978-953-307-556-3

Hard cover, 376 pages

Publisher InTech

Published online 01, August, 2011

Published in print edition August, 2011

Graphene, a conceptually new class of materials in condensed-matter physics, has been the interest of many theoretical studies due to the extraordinary thermal, mechanical and electrical properties for a long time. This book is a collection of the recent theoretical work on graphene from many experts, and will help readers to have a thorough and deep understanding in this fast developing field.

\title{
How to reference
}

In order to correctly reference this scholarly work, feel free to copy and paste the following:

L.L. Bonilla and A. Carpio (2011). Theory of Defect Dynamics in Graphene, Graphene Simulation, Prof. Jian Gong (Ed.), ISBN: 978-953-307-556-3, InTech, Available from: http://www.intechopen.com/books/graphenesimulation/theory-of-defect-dynamics-in-graphene

\section{INTECH}

open science | open minds

\section{InTech Europe}

University Campus STeP Ri

Slavka Krautzeka 83/A

51000 Rijeka, Croatia

Phone: +385 (51) 770447

Fax: +385 (51) 686166

www.intechopen.com

\section{InTech China}

Unit 405, Office Block, Hotel Equatorial Shanghai

No.65, Yan An Road (West), Shanghai, 200040, China

中国上海市延安西路65号上海国际贵都大饭店办公楼405单元

Phone: +86-21-62489820

Fax: $+86-21-62489821$ 
(C) 2011 The Author(s). Licensee IntechOpen. This chapter is distributed under the terms of the Creative Commons Attribution-NonCommercialShareAlike-3.0 License, which permits use, distribution and reproduction for non-commercial purposes, provided the original is properly cited and derivative works building on this content are distributed under the same license. 